\title{
Large Exciton Diffusion Coefficients in Two Dimensional Covalent Organic Frameworks with Different Domain Sizes Revealed by Ultrafast Exciton Dynamics
}

\author{
Nathan C. Flanders, ${ }^{1}$ Matthew S. Kirschner, ${ }^{1}$ Pyosang Kim, ${ }^{1}$ Austin M. Evans, ${ }^{1}$ \\ Thomas J. Fauvell, ${ }^{1,3}$ Waleed Helweh, ${ }^{1}$ Austin P. Spencer, ${ }^{1}$ Richard D. Schaller, ${ }^{1,2}$ \\ William R. Dichtel, ${ }^{1}$ and Lin X. Chen ${ }^{1,3}$ \\ ${ }^{1}$ Department of Chemistry, Northwestern University, \\ 2145 Sheridan Road, Evanston, IL, 60208 USA \\ ${ }^{2}$ Center for Nanoscale Materials, Argonne National Laboratory, \\ Argonne, IL 60439, USA. \\ ${ }^{3}$ Chemical Sciences and Engineering Division, Argonne National Laboratory, \\ Argonne, IL 60439, USA.
}

\section{Supplementary Information}

\begin{tabular}{|c|}
\hline Correspondence Address \\
\hline Professor William R. Dichtel \\
Department of Chemistry \\
Northwestern University \\
2045 Sheridan Road \\
Evanston, IL 60208 (USA) \\
wdichtel@northwestern.edu \\
Professor Lin X. Chen \\
Department of Chemistry \\
Northwestern University \\
2045 Sheridan Road \\
Evanston, IL 60208 (USA) \\
1-chen@northwestern.edu \\
\hline
\end{tabular}

Table of Contents
A. Supplementary Note 1
S-2
B. Additional Characterization
S-2
C. References
S-8 
Supplementary note 1: Determination of Exciton delocalization and coupling strength of Exciton in COF-5. As characterized by Spano and coworkers, the ratio of vibronic peaks in emission spectrum can be used to characterize the extent of coupling strength in aggregates. The Coherence length can be described by

$N_{C o h}=\frac{1}{\left\langle C^{(e m)}(0)\right\rangle} \sum_{s}\left|\left\langle C^{(e m)}(s)\right\rangle_{C}\right|$

And using the relationship between the relative ratios of vibronic bands as employed by Spano yields the equation:

$$
N_{C o h} \approx \lambda^{2} \frac{\left\langle I^{0-0}\right\rangle_{C}}{\left\langle I^{0-1}\right\rangle_{C}}
$$

The ratio of the $0-0$ to $0-1$ in molecular HHTP was 1.9 , in close agreement with the predicted 2 based on expected Franck-Condon Progression. However, upon condensation into the COF the ratio changed to 1 . Since in the molecular species $N_{C o h}$ is assumed to be 1 , the $N_{C o h}$ for the COF can be calculated to be 2 based on the ratio of the molecular intensity ratios to those found in the COF. ${ }^{1-3}$

\section{B. Additional Characterization}

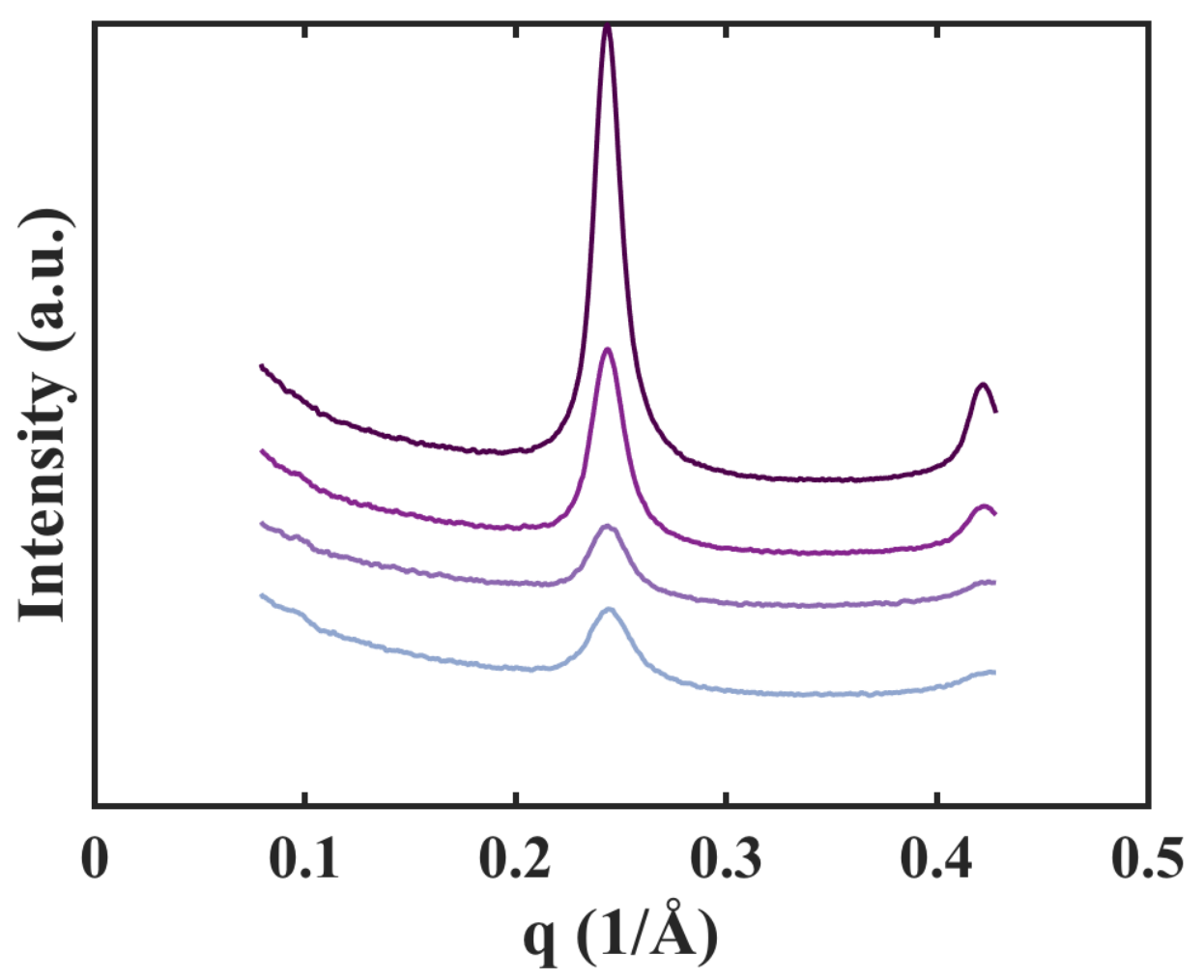

Figure S1. XRD profile of (100) and (110 peaks) 


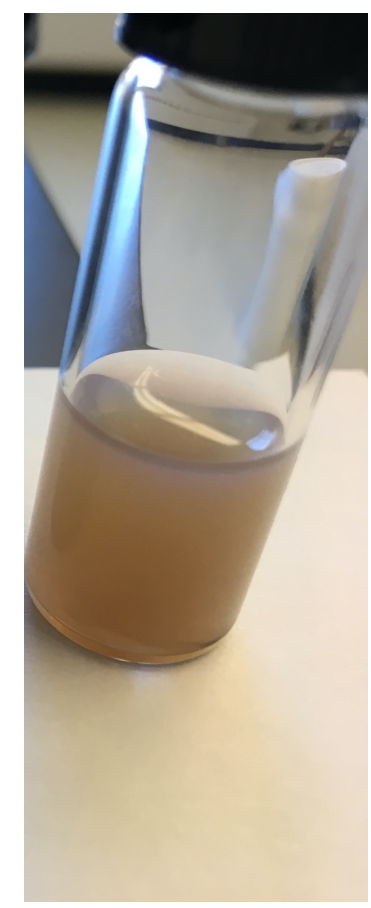

Figure S2. Colloidal suspension of COF-5

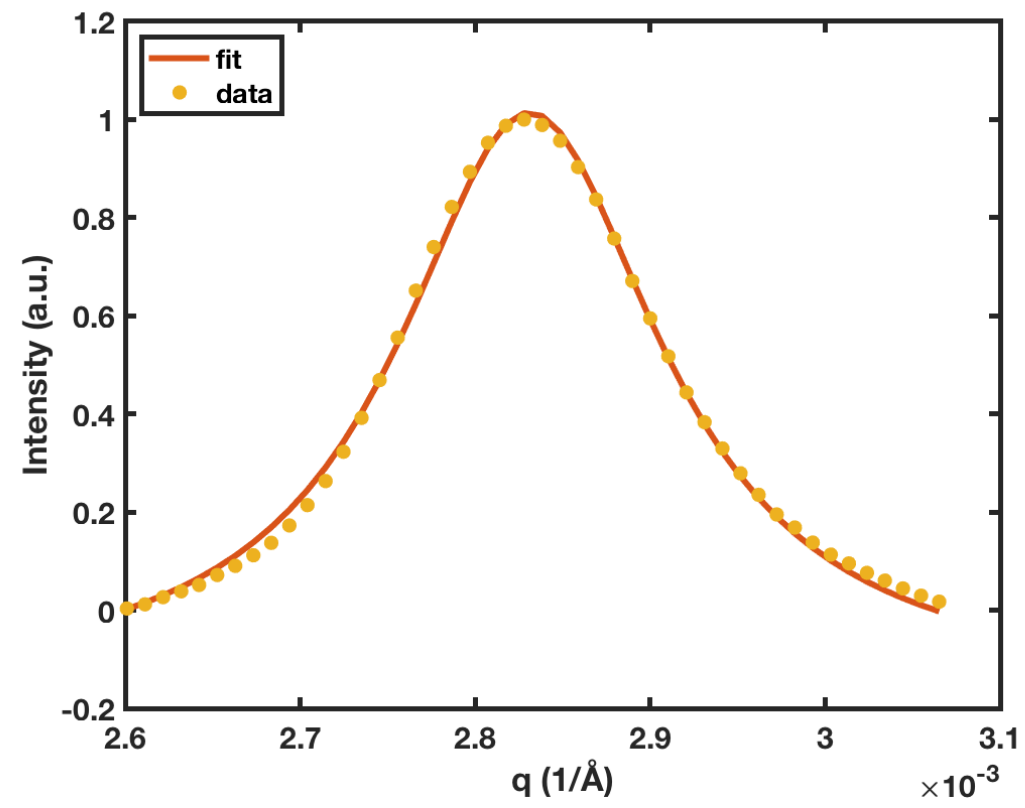

Figure S3. (100) Bragg diffraction peak fit to Lorentzian lineshape function to characterize domain size of COF nanocrystal via Scherrer equation. ${ }^{4}$ 


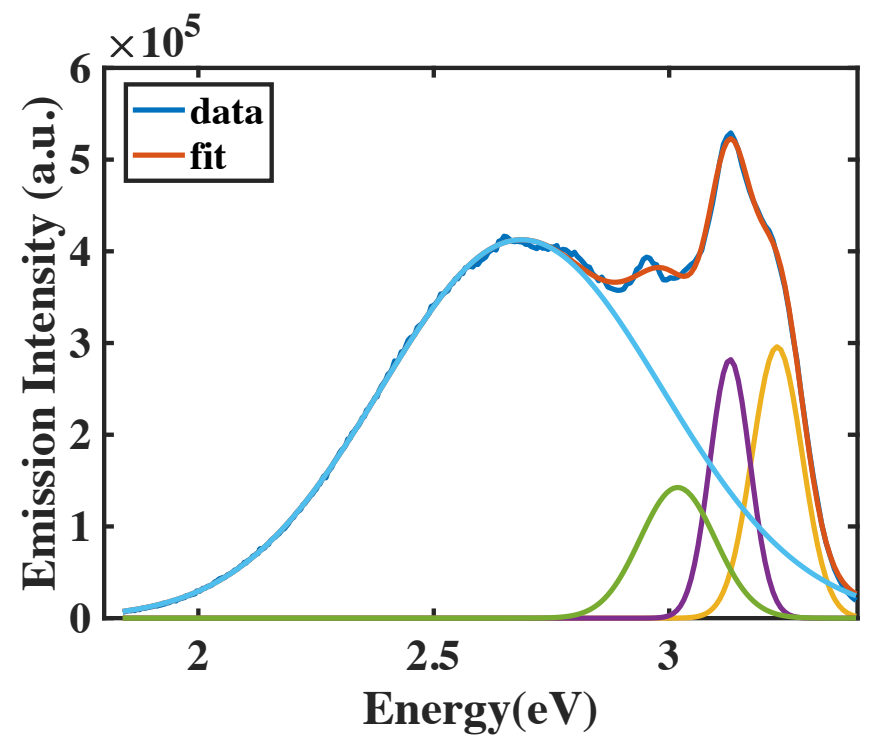

Figure S4. Result of 4 Gaussian fittings on emission spectra of different emission components as fit to aforementioned coherence function to reveal delocalization length. 0-0 transition fit is displayed as yellow and 0-1 as purple

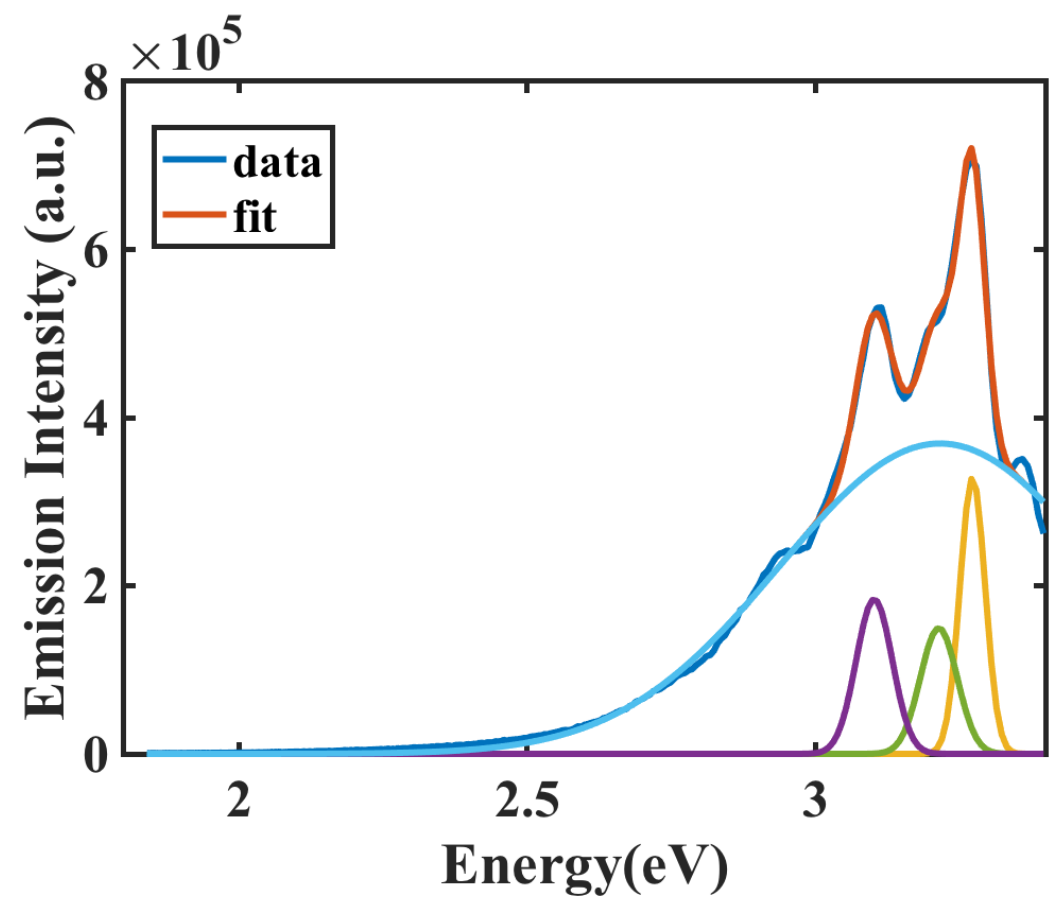

Figure S5. Result of 4 Gaussian fittings on emission spectra of Molecular HHTP as fit to aforementioned coherence function to reveal delocalization length. 0-0 transition fit is displayed as yellow and 0-1 as purple. 


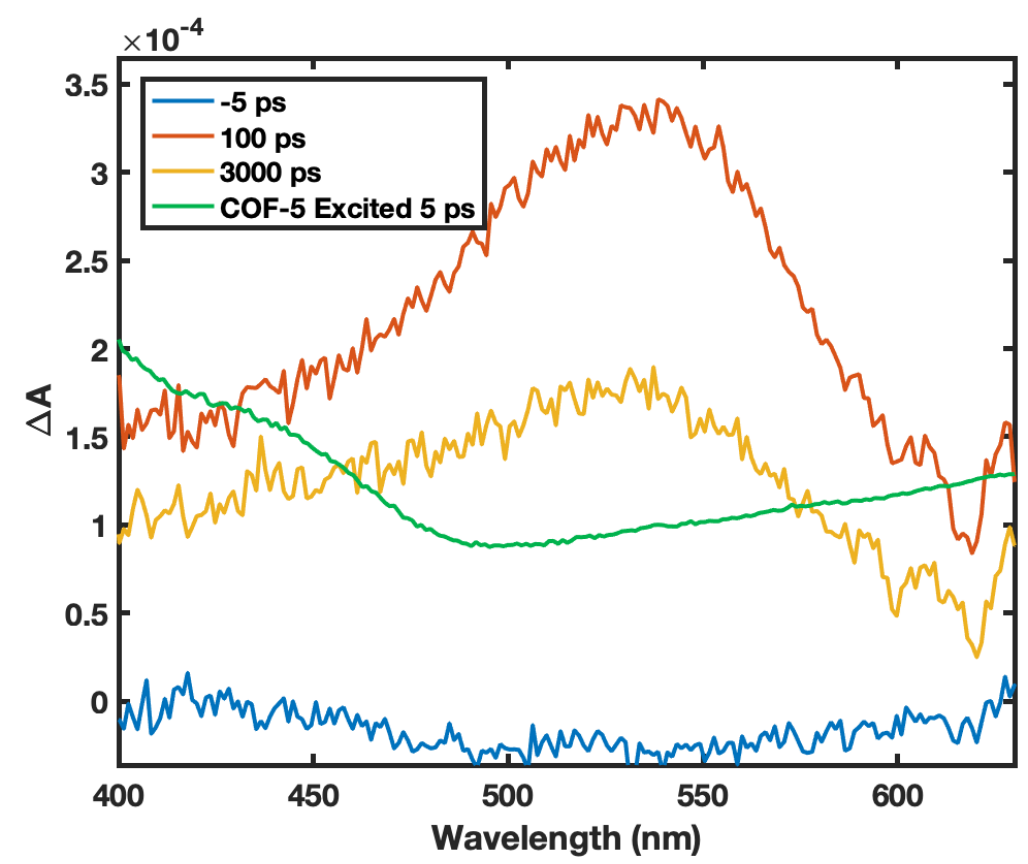

Figure S6. HHTP excited state absorption plotted with COF-5 excited state absorption to reveal differences in spectra.

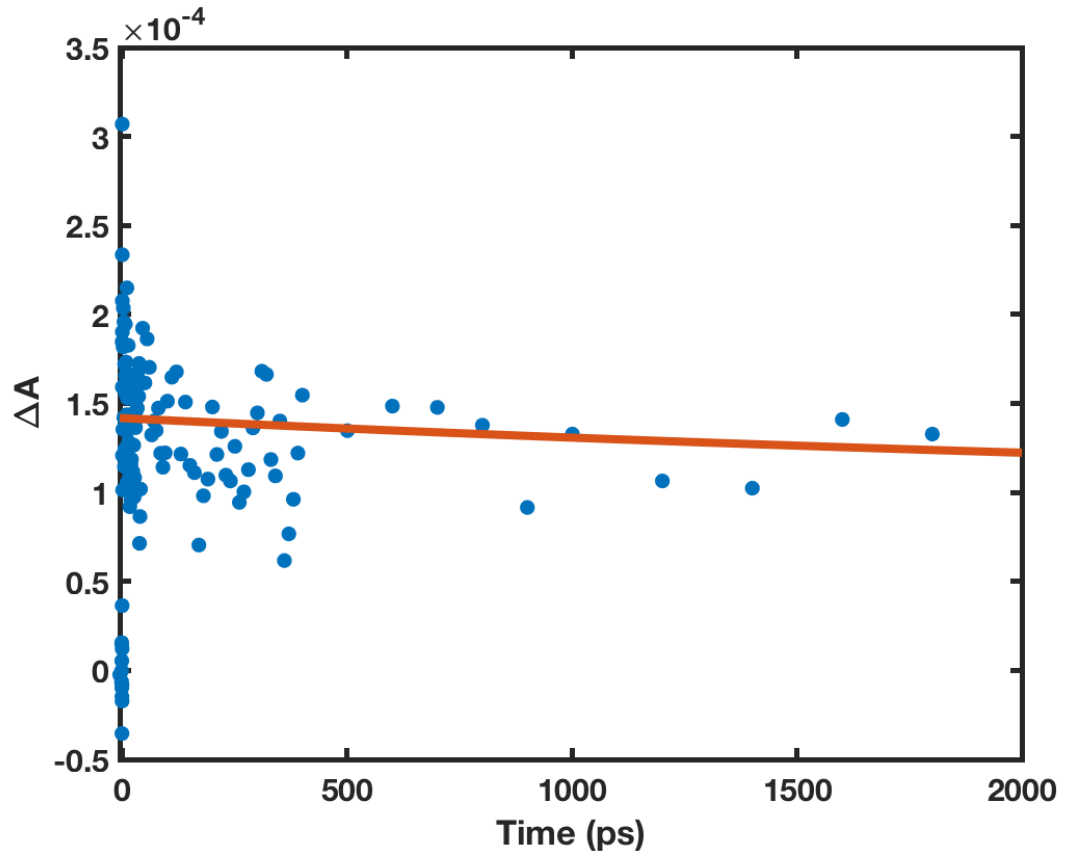

Figure S7. HHTP kinetic trace with kinetics unresolvable for duration of experiment 


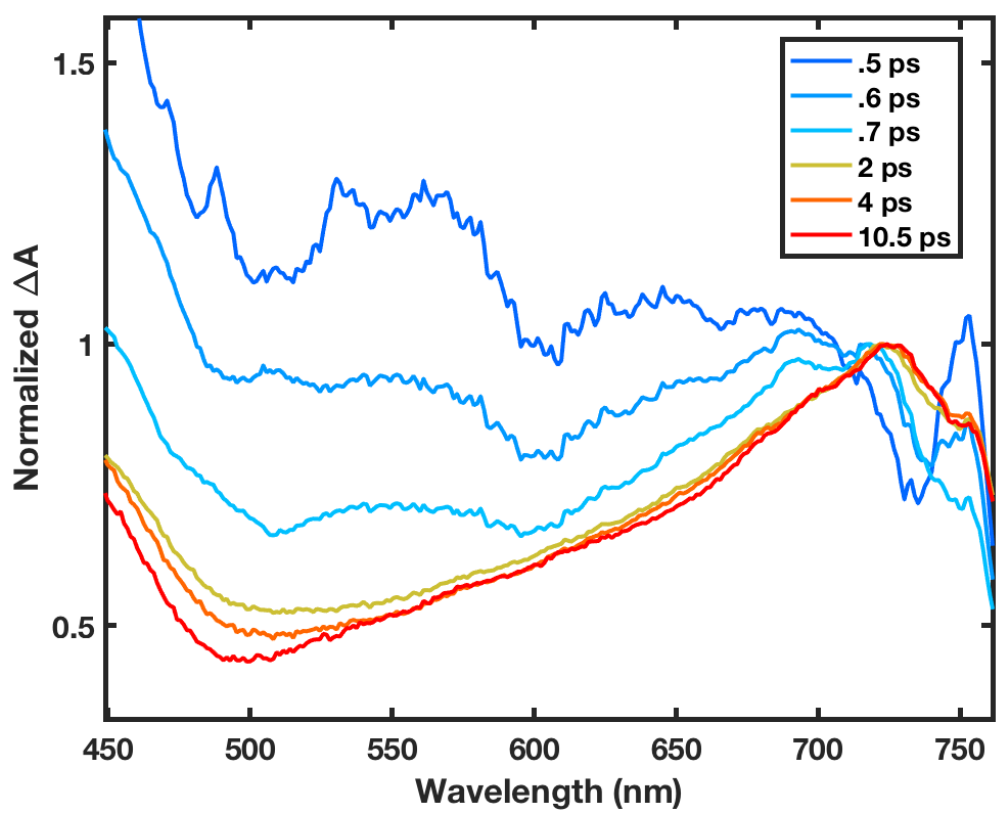

Figure S8. COF-5 transient absorption spectra normalized to far red ESA feature revealing emergence of SE feature.

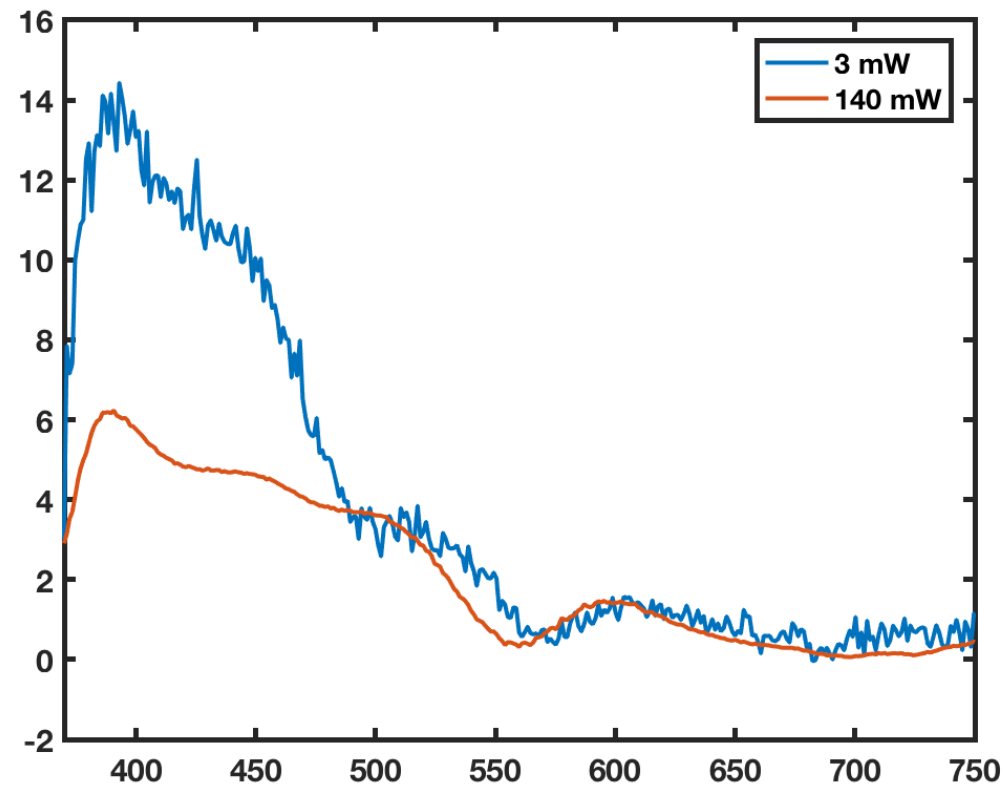

Figure S9. Two different power COF-5 spectra at 1.5 ps normalized, revealing divergent absorption for far blue feature confirming loss of singlet population at high fluences. 


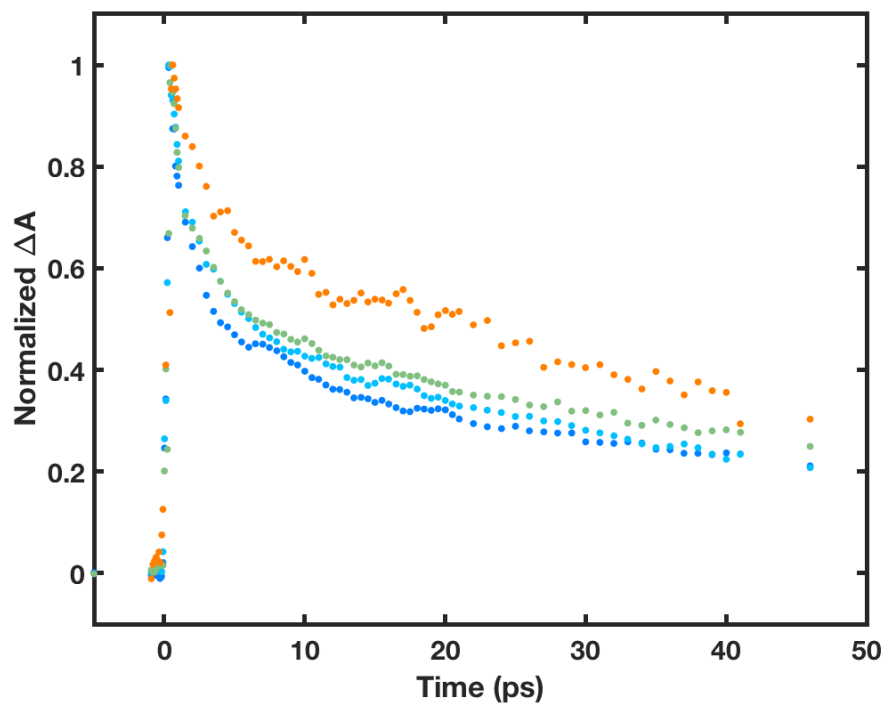

Figure S10. Kinetic traces of different size COF $5(36 \mathrm{~nm}$ - orange, $29 \mathrm{~nm}$ - green, $24 \mathrm{~nm}-$ light blue, $22 \mathrm{~nm}$ - dark blue) following photoexcitation at high fluence observed at $400 \mathrm{~nm}$ without offset subtraction.

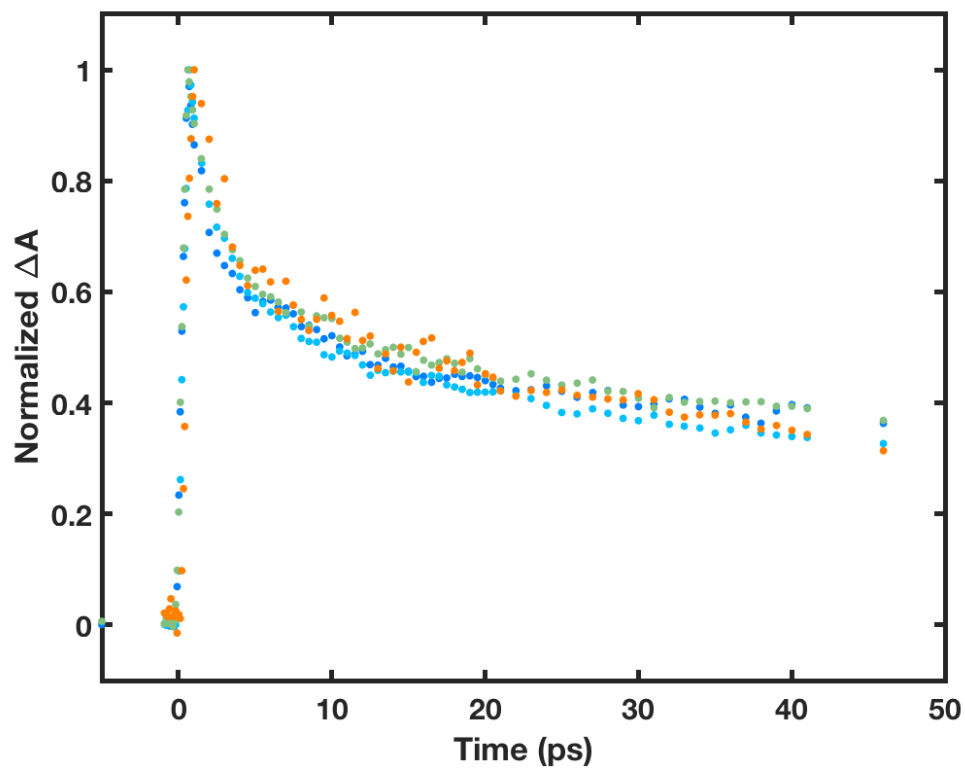

Figure S11. Kinetic traces of different size COF $5(36 \mathrm{~nm}$ - orange, $29 \mathrm{~nm}$ - green, $24 \mathrm{~nm}-$ light blue, $22 \mathrm{~nm}$ - dark blue) following photoexcitation at high fluence observed at $650 \mathrm{~nm}$ revealing non divergent kinetics. 


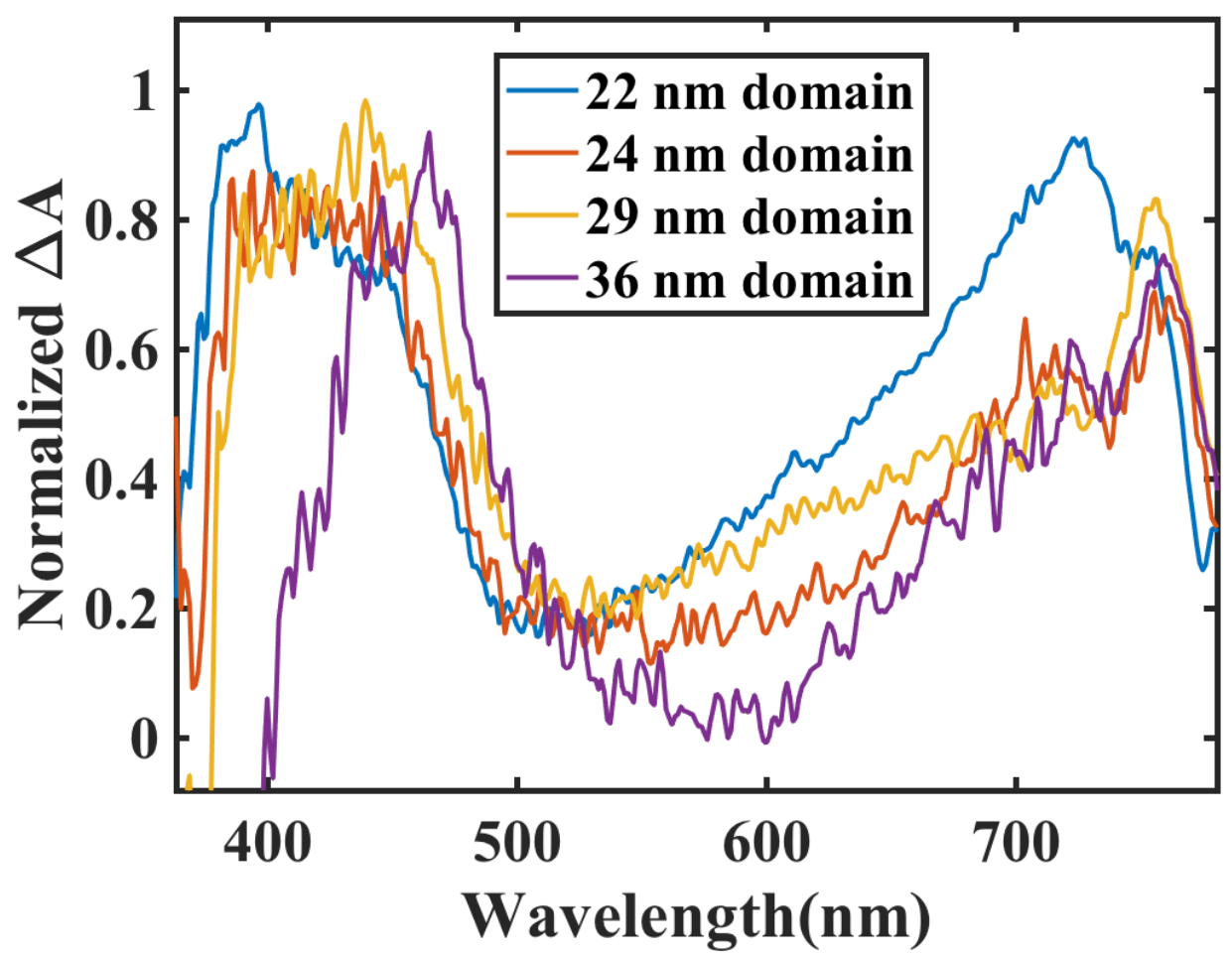

Figure S12. Excited state absorption of different size COF-5 colloid domains observed at 3 ps revealing redshift of blue ESA feature with size.

\section{References}

(1) Sung, J.; Kim, P.; Fimmel, B.; Würthner, F.; Kim, D. Direct Observation of Ultrafast Coherent Exciton Dynamics in Helical $\pi$-Stacks of Self-Assembled Perylene Bisimides. Nat. Commun. 2015, 6. https://doi.org/10.1038/ncomms9646.

(2) Spano, F. C. The Spectral Signatures of Frenkel Polarons in H-and J-Aggregates. Acc. Chem. Res. 2009, 43 (3), 429-439. https://doi.org/10.1021/ar900233v.

(3) Spano, F. C.; Clark, J.; Silva, C.; Friend, R. H. Determining Exciton Coherence from the Photoluminescence Spectral Line Shape in Poly ( 3-Hexylthiophene) Thin Films Shape in Poly , 3-Hexylthiophene ... Thin Films. J. Chem. Phys. 2009, 130 (074904). https://doi.org/10.1063/1.3076079.

(4) Patterson, A. L. The Scherrer Formula for X-Ray Particle Size Determination. Phys. Rev. B 1939, 56, 978-982. 